\title{
Antimicrobial Susceptibility Patterns of Salmonella Species from Sources in Poultry Production Settings in Calabar, Cross River State, Nigeria
}

\author{
Nchawa Yangkam Yhiler ${ }^{1}$, Bassey Enya Bassey ${ }^{1,2, ~ *}$ \\ ${ }^{1}$ Department of Medical Laboratory Science, Faculty of Allied Medical Science, University of Calabar, Calabar, Nigeria \\ ${ }^{2}$ World Health Organization, UN House, Central Area District, FCT, Abuja, Nigeria
}

Email address:

yankgam@yahoo.com (N. Y. Yhiler), bassey69@yahoo.com (B. E. Bassey)

\section{To cite this article:}

Nchawa Yangkam Yhiler, Bassey Enya Bassey. Antimicrobial Susceptibility Patterns of Salmonella Species from Sources in Poultry Production Settings in Calabar, Cross River State, Nigeria. American Journal of Health Research. Vol. 3, No. 2, 2015, pp. 76-81.

doi: 10.11648/j.ajhr.20150302.14

\begin{abstract}
Globalisation and technology especially in the area of antimicrobial chemotherapy have greatly improved the quality of health care in both the veterinary and the public settings. Nonetheless, irrational use of antibiotics has considerably encouraged the development of antimicrobial resistance. This present study was aimed at determining the sources within the poultry production setting that are significantly responsible in the development and spread of antibiotic resistant strains of Salmonella. A total of 374 samples were collected from poultry environment sources (170), from poultry bird themselves (136) and from poultry personnel sources (68) in Calabar within the period of August 2013 and May 2014. The isolation of Salmonella was in accordance with the ISO 6579:2002 and the antibiotic susceptibility testing was carried out based on the CLSI by means of the Kirby-Bauer disk diffusion method involving 12 antibiotic disks (HDx). The paired sample t-test was used to determine the significant differences in the resistance of Salmonella species from the poultry sources against the antibiotics used in this study at $95 \%$ confidence level. The Salmonella isolates exhibited the highest rate of resistance against Ampicilin-10 $\mu \mathrm{g}(72.9 \%)$ and the least against Gentamicin-10 $\mu \mathrm{g}(1.4 \%)$. All the Salmonella isolates were sensitive to Chloramphenicol-30, Ciprofloxacine-5 $\mu \mathrm{g}$ and Imipenem-10 $\mu \mathrm{g}$ and hence the most sensitive. Majority (68.8\%) exhibited resistance against more than one type of antibiotics hence revealing a high rate of multidrug resistant Salmonella strains. The results of the paired sample t-test revealed that the development and spread of resistance against antibiotics was significantly dependent on the poultry environment sources and therefore should serve as important targets for prevention measures.
\end{abstract}

Keywords: Salmonella, Antibiotic Susceptibility, Multidrug Resistance

\section{Introduction}

Salmonella serotypes have been extensively incriminated as the most important zoonotic pathogens in several countries worldwide [1]. They are responsible for the significant morbidity and mortality in both humans and animals [1]. Each year, an estimated 1.3 billion cases resulting to about 3 million deaths occur worldwide due to salmonellosis alone [2,3]. In spite of the importance of poultry as the major element in the human food chain, it has been frequently labelled as one of the most important sources of food poisoning due to Salmonella serovars causing the majority of food born outbreaks worldwide $[4,3,5,6,7,8,9]$.

The use of antibiotics together with the improvement of sanitation and hygiene as well as immunization and proper nutrition has provided major benefits in human life expectancy [10]. However, the increased utilization of antibiotics in both public and veterinary settings has led to the emergence of antibiotic resistance and as a consequence poses a serious threat to public health safety [10]. In general, the persistent use of drugs initiates selective pressure that encourages the development of antibiotic resistant pathogens. One of the most important factors necessary for the development of antibiotic resistant strains of microorganisms is the irrational use of such antibiotics in the animal production settings, which has brought about the emergence of bacteria strains that were otherwise infective only to animals to now become infective to humans as well due to the acquisition of the antibiotic resistance traits [3].

The drastic and continuous increase in the development of 
drug resistant Salmonella strains in recent years has been frequently reported and is of major concern in both the developed and the developing countries [11, 12]. Animals that have become infected with antibiotic resistant strains of Salmonella are important sources of resistant determinants that give room for the Salmonella serovars to become infective to humans [5]. Antibiotic resistant strains of Salmonella have been frequently recovered from food of animal origin of which poultry is of major concern $[13,14]$. In veterinary medicine, the use of antimicrobial agents is exploited in animal feeds as prophylaxis and as growth promoters in order to enhance growth and to improve the health of the animals. However, antibiotic incorporated into animal feeds are at sub-therapeutic concentrations which in consequence creates a favourable ground for the development of antibiotic resistance within the animal production setting [15].

The frequency of isolation of Salmonella strains resistant to several antimicrobial agents has increased in several countries worldwide [16, 17] and the association between the veterinary use of antibiotics and the development of resistant strains of microorganisms against these antibiotics in human infection is now an established fact [18]. Multidrug resistance against commonly used antibiotics in both the veterinary and the public setting have been found to be exhibited by almost all Salmonella serotypes [11].

In spite of the relatively considerable information of antimicrobial susceptibility of Salmonella serotypes in human infections and the contamination of foods of animal origin in many parts of the world, there is however a dearth of such information in most parts of Africa including Nigeria [19]. Therefore, it is of importance to monitor antimicrobial resistance of Salmonella serovars from the production setting of food animals of which poultry is a major concern. This study therefore attempts to analyse the antibiotic resistance of Salmonella serovars recovered from three major sources (the poultry environment, the poultry birds themselves and the poultry personnel) in the poultry production setting in Calabar.

\section{Materials and Methods}

\subsection{Study Design and Sample Collection}

This study was carried out within the periods of August 2013 and May 2014 in the Calabar metropolis of Nigeria. Three hundred and seventy four (374) specimens were randomly collected from three different sources within the poultry production settings in Calabar; out of which 170 were obtained from the poultry environmental sources which included poultry feeds, poultry drinking water, poultry litter, poultry abattoir reins and dust from poultry house; 136 were obtained from poultry birds themselves which included guts, cloacal swabs, poultry meat and eggs; and 68 were obtained from the poultry personnel which included the poultry personnel stool and hand washings.

Samples were collected based on the method as described by [20]. The samples were immediately placed in an insulating box containing ice packs in order to maintain the temperature at $4-6^{\circ} \mathrm{C}$ and transported to the laboratory within four hours.

\subsection{Isolation and Identification of Salmonella Species}

The isolation of Salmonella species from the poultry production setting in Calabar was based on the ISO 6579:2002 involving the non-selective pre-enrichment stage using 10\% buffered Peptone Water, the selective enrichment stage using Modified Semisolid Rapapport-Vassiliadis and Muller-Kauffmann Tetrathionate-nivobiocin broths, and finally the selective plating stage using Brilliant Green and Xylulose Lysine Deoxycholate agars. Typical Salmonella colonies on Brilliant Green and Xylulose Lysine Deoxycholate agars were purified by further sub-culturing on Nutrient agar prior to confirmation.

\subsection{Confirmation of the Salmonella Isolates}

The presumptive Salmonella isolates were confirmed following their morphological, biochemical and serological characteristics. The morphological characteristics included their colonial appearance on Brilliant Green and Xylulose Lysine Deoxycholate agar plates as well as their Gram reaction when observed under the $100 \mathrm{X}$ objective of a light microscope. The biochemical reaction included their characteristics on Triple Sugar Iron agar slants, Christensen agar slats, urea, Lysine decarboxylase test, $\beta$-galactosidase test, Acetone production test, indole test and methyl red test (Hardy Diagnostics, CA, USA). Finally, confirmation was supplemented by serology using commercially available polyvalent Salmonella serum (Denka Sieken Co. Ltd. Japan) specific against all group and type factor Salmonella antigens. Those colonies that agglutinated within two minutes were confirmed as belonging to the genus Salmonella. They were then stored on Nutrient agar slants overlaid with paraffin oil at $4^{0} \mathrm{C}$ prior to antibiotic susceptibility testing.

\subsection{Antibiotic Susceptibility Testing}

The antimicrobial susceptibility testing was carried out based on the Clinical Laboratory Standard Institute (CLSI) method by means of the Kirby-Bauer Disk Diffusion test using Mueller-Hinton agar (Hardy Diagnostics CA, USA) [21].

Four to five colonies of each isolate were transferred into a test tube containing $2.5 \mathrm{ml}$ of sterile normal saline by means of a sterile wire loop. The suspension was vortexed and the turbidity compared with that of Barium Chloride (0.5 McFarland Turbidity Standard: $\left.1.0 \times 10^{8} \mathrm{CFU} / \mu \mathrm{l}\right)$. The optical density of the standard was regularly monitored by means of a spectrophotometer at $\lambda=625 \mathrm{~nm}$ and $1 \mathrm{~cm}$ light path $(\mathrm{OD} \lambda=0.08-0.1)$ [22]. $100 \mu \mathrm{l}$ of the bacterial suspension was then inoculated on the iso-sensitivity test agar plates, and the excess was siphoned by means of sterile Pasteur pipettes. The plates were allowed to dry at room temperature in a laminar flow. The pre-determined antibiotic 
disks were then dispensed into the bacterial lawn by means of a sterile pair of forceps and gently pressed to ensure complete contact with the agar. The discs were positioned $15 \mathrm{~mm}$ way from the edge of the plate and $25 \mathrm{~mm}$ away from each other. The plates were incubated at $35-37^{\circ} \mathrm{C}$ for 18 to $24 \mathrm{hrs}$. The diameters of the zones of inhibition were read and interpreted in accordance with [21]. The antibiotic disks used in this study included 12 commercially available drug disks (Hardy Disks, HDx): cefotaxime-30 $\mu \mathrm{g}$ (CTX), ceftazidime - $30 \mu \mathrm{g}$ (CAZ), chloramphenicol - $30 \mu \mathrm{g}(\mathrm{CH})$, ciprofloxacin $-5 \mu \mathrm{g}$ (CIP), carbenicillin $100 \mu \mathrm{g}$ (CB), amoxicillin/clavulanic acid-30 $\mu \mathrm{g}(\mathrm{AmC})$, ampicillin $10 \mu \mathrm{g}$ (AM), amikacin -30 $\mu \mathrm{g}(\mathrm{AN}), \quad$ tetracycline-30 $\quad \mu \mathrm{g} \quad$ (TE), sufamethaxole/trimethoprime - $25 \mu \mathrm{g}$ (SXT), imipenem - 10 $\mu \mathrm{g}$ (IMP) and gentamicin - $10 \mu \mathrm{g}$ (GE).

\subsection{Statistical Analysis}

The data generated in this study were analysed using the predictive analytic software (PASW) 18.0 (IBM, USA). The paired sample student t-test was used to determine significant differences in the resistance of Salmonella species isolated from the poultry environmental, bird, and personnel sources in the poultry production system against the antibiotics used in this study. $\mathrm{P}$ values of less than $0.05(\mathrm{P}<0.05)$ was considered statistically significant.

\section{Results}

Out of the 374 specimens obtained from the poultry production setting in Calabar, 221 (59.1\%) were found to be positive for Salmonella species as determined by the morphological, biochemical and serological confirmation characteristics. Salmonella species were recovered from all the three sources in the poultry production setting. Samples from the poultry environmental sources revealed $58.8 \%$ rate of recovery of Salmonella species, $55.1 \%$ from poultry bird source and $67.6 \%$ from poultry personnel sources.

The antibiotic susceptibility testing revealed that the Salmonella isolates exhibited the highest resistance against ampicillin - $10 \mu \mathrm{g}(72.9 \%)$ and the least resistance against gentamicin - $10 \mu \mathrm{g}$ (1.4\%). However SXT, CTX, TE, AmC, $\mathrm{CB}, \mathrm{CAZ}$, and AN exhibited 46.2\%, 44.4\%, 38.0\%, 29.4\%, $20.4 \%, 19.5 \%$ and $5.0 \%$ resistance respectively. All the Salmonella isolates exhibited zero resistance (sensitive) to chloramphenicol - $30 \mu \mathrm{g}$, ciprofloxacin - $5 \mu \mathrm{g}$ and imipenem $-10 \mu \mathrm{g}$.

With respect to the resistance of the Salmonella isolates against the different antibiotics used in this study, the Salmonella isolates were grouped thus: those that exhibited zero resistance (were sensitive) to all the antibiotics used (Stypes), those that exhibited resistance against only one type of antibiotic disk (Single R-type), those that exhibited resistance against more than one type of antibiotic disk (multiple R-type).

The majority of all the Salmonella isolates were of the multiple R-type $(68.8 \%)$, with varied resistance patterns. Only a very few of the Salmonella isolates $9.5 \%$ were of the S-types while $21.7 \%$ of the Salmonella isolates were of the single R-type.

The Salmonella isolates recovered from the poultry environmental sources showed the highest percentage of multiple R-types (78.0\%) involving 9 antibiotic disc types out of 12 (AM, SXT, CTX, TE, AmC, CB, CAZ, AN and GE) followed by the Salmonella isolates from the poultry bird sources $(65.3 \%)$ involving 7 out of 12 antibiotic disk types (AM, SXT, CTX, TE, AmC, CB and CAZ) and then the Salmonella isolates from the poultry personnel sources (54.3\%) involving 8 out of 12 antibiotic disc types (AM, SXT, CTX, TE, AmC, CB, CAZ and AN). However, the Salmonella isolates from all three sources were resistant to at least 7 out of 12 antibiotics (AM, SXT, CTX, TE, AmC, CB and CAZ).

Table 1. Frequency of Salmonella species recovered from sources in the poultry production settings.

\begin{tabular}{llll}
\hline Poultry Source & Number collected & Number Positive & Percentage Positive (\%) \\
\hline Environment & 170 & 100 & 58.8 \\
Bird & 136 & 75 & 55.1 \\
Personnel & 68 & 46 & 67.6 \\
Total & 374 & 221 & 59.1 \\
\hline
\end{tabular}

Table 2. Resistance pattern of Salmonella species isolated from poultry sources against different antibiotics.

\begin{tabular}{|c|c|c|c|c|}
\hline \multirow{2}{*}{ Antibiotic disks } & \multicolumn{3}{|c|}{ Resistance of Salmonella isolates from sources } & \multirow{2}{*}{ Total } \\
\hline & Environmental (\%). $(\mathrm{n}=100)$ & Bird (\%). $(n=75)$ & Personnel $(\%) \cdot(n=46)$ & \\
\hline Ampicillin $10 \mu \mathrm{g}(\mathrm{AM})$ & $74(74.0)$ & $54(72.0)$ & $33(71.7)$ & $161(72.9)$ \\
\hline Sufamethaxole/ trimethoprime - $25 \mu \mathrm{g}$ (SXT) & $51(51.0)$ & $33(44.0)$ & $18(39.1)$ & $102(46.2)$ \\
\hline Cefotaxime $-30 \mu \mathrm{g}(\mathrm{CTX})$ & $49(49.0)$ & $24(32.0)$ & $25(54.3)$ & $98(44.4)$ \\
\hline Tetracycline - $30 \mu \mathrm{g}$ (TE) & $37(37.0)$ & $23(30.7)$ & $24(52.2)$ & $84(38.0)$ \\
\hline Amoxicillin/clavulanic acid - $30 \mu \mathrm{g}$ (AmC) & $34(34.0)$ & $18(24.0)$ & $13(28.3)$ & $65(29.4)$ \\
\hline Carbenicillin $100 \mu \mathrm{g}(\mathrm{CB})$ & $24(24.0)$ & $10(13.0)$ & $11(23.9)$ & $45(20.4)$ \\
\hline Amikacin $30 \mu \mathrm{g}(\mathrm{AN})$ & $6(6.0)$ & $0(0.0)$ & $5(10.9)$ & $11(5.0)$ \\
\hline Gentamicin - $10 \mu \mathrm{g}$ (GE) & $3(3.3)$ & $0(0.0)$ & $0(0.0)$ & $3(1.4)$ \\
\hline Ciprofloxacine - $5 \mu \mathrm{g}$ (CIP) & $0(0.0)$ & $0(0.0)$ & $0(0.0)$ & $0(0.0)$ \\
\hline Imipenem - $10 \mu \mathrm{g}$ (IMP) & $0(0.0)$ & $0(0.0)$ & $0(0.0)$ & $0(0.0)$ \\
\hline Chloramphenicole - $30 \mu \mathrm{g}(\mathrm{Ch})$ & $0(0.0)$ & $0(0.0)$ & $0(0.0)$ & $0(0.0)$ \\
\hline
\end{tabular}


Table 3. Antibiotic disk types involved in Salmonella resistance in the poultry production setting.

\begin{tabular}{llllll}
\hline $\begin{array}{l}\text { Source of Salmonella } \\
\text { isolates }\end{array}$ & $\begin{array}{l}\text { Total number of } \\
\text { Salmonella } \text { isolates }\end{array}$ & $\begin{array}{l}\text { Number of } \\
\text { S-type (\%) }\end{array}$ & $\begin{array}{l}\text { Number of single R- } \\
\text { type }\end{array}$ & $\begin{array}{l}\text { Number of multiple } \\
\text { R-type }\end{array}$ & $\begin{array}{l}\text { Antibiotic disks } \\
\text { involved in } \\
\text { resistance }\end{array}$ \\
\hline $\begin{array}{l}\text { Poultry environmental } \\
\text { sources }\end{array}$ & 100 & $5(5.0)$ & $17(17.0)$ & $78(78.0)$ & $\begin{array}{l}\text { AM, SXT, CTX, TE, } \\
\text { AmC, CB, CAZ, AN } \\
\text { and GE } \\
\text { AM, SXT, CTX, TE, } \\
\text { AmC, CB and CAZ } \\
\text { Poultry bird sources }\end{array}$ \\
$\begin{array}{lllll}\text { Poultry personnel sources } \\
\text { Total }\end{array}$ & 46 & $9(12.0)$ & $17(22.7)$ & $49(65.3)$ & $\begin{array}{l}\text { AM, SXT, CTX, TE, } \\
\text { AmC, CB, CAZ and } \\
\text { AN } \\
\text { AM, SXT, CTX, TE, } \\
\text { AmC, CB and CAZ }\end{array}$ \\
\hline
\end{tabular}

S-type: Salmonella isolates that exhibited zero resistance (sensitive) to all the antibiotics used

Single R-type: Salmonella isolates that exhibited resistance to a single type of antibiotic disk type

Multiple R-type: Salmonella isolates that exhibited resistance to more than one antibiotic disk type

Table 4. Statistical comparison of the mean resistance of Salmonella isolates from the poultry personnel, bird and environmental sources in the poultry production setting against different antibiotics.

\begin{tabular}{|c|c|c|c|c|c|}
\hline Pairs & Standard deviation & $\mathbf{t}$ & df & P - value & Remark \\
\hline Bird sources vs. Personnel sources & 7.5959 & 1.216 & 11 & 0.249 & $\begin{array}{l}\mathrm{P}>0.05 \text { : } \\
\text { Not statistically significant }\end{array}$ \\
\hline Environmental sources vs. Personnel sources & 14.2667 & 3.055 & 11 & 0.011 & $\begin{array}{l}\mathrm{P}<0.05: \\
\text { Statistically significant }\end{array}$ \\
\hline Environmental sources vs. Bird sources & 8.8980 & 4.2632 & 11 & 0.003 & $\begin{array}{l}\mathrm{P}<0.05: \\
\text { Statistically significant }\end{array}$ \\
\hline
\end{tabular}

There was no statistical significant difference in resistance of Salmonella isolates from the poultry personnel sources when compared with the mean resistance of those from the poultry bird sources against the antibiotics used $(\mathrm{P}=0.249)$. There was a statistical significant difference in the resistance of the Salmonella isolates from the poultry personnel sources against the antibiotics used compared with the mean resistance of those from the poultry environmental sources against the antibiotics used $(\mathrm{P}=0.011)$. Also, there was a statistical significant difference in the resistance of Salmonella isolates from the poultry bird sources compared with the mean resistance of those from the poultry environmental sources against the antibiotics used $(\mathrm{P}=$ 0.003).

\section{Discussion}

Recently, there has been a marked increase of in the development of antimicrobial resistant strains of Salmonella from foods of animal origin especially poultry. Antimicrobial resistant strains of Salmonella species from food animals are important in causing human infections which previously could not.

Non-typhoidal Salmonella species have been recognized as the major cause of food borne diseases posing major public health problems worldwide $[23,24]$. In humans, the disease is usually self-limiting when confined within the intestines, even though sometimes it may result to significant discomforts. In some cases however, the infection may cross the intestinal barrier to reach other vital organs resulting in serious complications in which case require significant antimicrobial interventions [17]. This therefore calls for the surveillance and sharing of antimicrobial susceptibility data especially in the poultry production setting in order to ensure the effective implementation of public health control programs.

Multidrug resistance is phenomenal when resistance is observed against classical first line antimicrobial agents like ampicillin and sulfamethaxole/timethoprime [25] and this implies that the Salmonella isolates in the poultry production setting in Calabar exhibited multidrug resistance. The results of this study corroborates [6] who demonstrated the resistance of Salmonella isolates against commonly used antimicrobials in the poultry production setting in Edo-Ekiti, Nigeria. Some workers demonstrated a high resistance of Salmonella against ampicillin (90.91\%) within the poultry production setting in the United Arab Emirates [26], and this is in close agreement with the result of this study.

The Salmonella isolates from the poultry production setting in Calabar exhibited resistance against 9 out of the 12 antibiotics used in this study. This reveals a wide range of antibiotics which the Salmonella species have become resistant against. Such a wide range of antibiotic resistance can be attributed to the gross indiscriminate use of antimicrobial agents in the poultry production setting [27, 28]. Furthermore, the incorporation of antibiotics in the diet of animals at sub therapeutic concentrations as prophylaxes and as growth promoters has invariably contributed to the development of antibiotic resistant strains of Salmonella [29]

This current study also revealed that all the Salmonella isolates recovered from the poultry production setting in Calabar were sensitive to Imipenem - $10 \mu \mathrm{g}$, Chloramphenicol - $30 \mu \mathrm{g}$ and Ciprofloxacin - $5 \mu \mathrm{g}$, implying these drugs to be the most active drugs against Salmonella 
species in the poultry production setting in Calabar and therefore could serve as the drugs of choice in the control of salmonellosis in the poultry production setting in Calabar. This closely agrees with the work carried out in Zaria, Nigeria [30], but is however contradicted by the work carried out in Cameroon [5]. Such differences could result from the difference in the geographical location as well as the habitual drug administration standards which differ with different regions [5].

Out of the 221 Salmonella isolates recovered from the poultry production setting, only $21(9.5 \%)$ were completely sensitive (no resistance) to all the 12 antibiotics used in this study whereas the rest $21.7 \%$ and $68.8 \%(90.5 \%)$ exhibited resistance against only a single antibiotic disk type and more than one antibiotic disc types respectively.

Based on the objective of this study, the null hypothesis stated that there is no significant difference in the resistance of Salmonella isolates from the different sources within the poultry production setting against all the antibiotics used in this study. However, when the resistance of the Salmonella species from the poultry personnel sources against the antibiotics used were compared with the resistance of those from the poultry bird sources against the antibiotics used, there was no statistical significant difference $(P=0.249)$. This implies that the resistance of the Salmonella species against all the antibiotics used was not dependent on either the poultry personnel sources or the poultry bird sources.

However, on the other hand, when the resistance of the Salmonella species isolated from the poultry environmental sources against the antibiotics used was compared with the resistance of those from the poultry personnel sources and the poultry bird sources against the antibiotics used, there was statistical significant difference in each of the cases $(\mathrm{P}=$ 0.011 and $\mathrm{P}=0.003$ respectively). This implies that the resistance of Salmonella species against the antibiotics used depended on the poultry environmental sources. Hence, the poultry environmental sources (poultry feeds, water, abattoir reins, litter from floor and dust from wall as used in this study) are important sources in the development of antibiotic resistant strains of Salmonella serving as a means of spread to the poultry birds, and the poultry personnel as well as the human population.

\section{Conclusion}

In conclusion, bacterial resistance against antibiotics greatly interferes with the effectiveness of control strategies against infectious diseases [31]. Therefore, good hygiene practices and the controlled use of antibiotics is necessary to prevent the spread of antibiotic resistant strains of Salmonella. This current study has revealed poultry environment sources as important sources of antibiotic resistant strains of Salmonella. This implies that in order for control strategies to be more effective in minimizing the development and spread of antibiotic resistant Salmonella strains, they should be geared towards preventing environmental contamination within the poultry production setting.

\section{References}

[1] Akinyemi KO, Phillip W, Beyer W, Bohm R. In -vitro antimicrobial susceptibility patterns of Salmonella enterica serovars and emergence of Salmonella phage type DT071 in a suspected community-associated outbreak in Lagos, Nigeria. $J$. Infect. Dev. Countries, 2007; 1(1): 48-54.

[2] P. L. Winokur, A. Brueggemann, D. L. DeSalvo, L. Hoffmann, M. D. Apley, E. K. Uhlenhopp, M. A. Pfaller, and G. V. Doern. Animal and human multidrug-resistant, cephalosporinresistant Salmonella isolates expressing a plasmid mediated CMY-2 AmpC beta-lactamase. Antimicrobial Agents of Chemotherapy 2000; 44: 2777-2783.

[3] Akond, M. A., Shirin M., Alam, S., Hassan, S. M. R., Rahman, M. \& Hoq M. Frequency of drug resistant Salmonellaspp. isolated from poultry samples in Bangladesh. Stamford Journal of Microbiology, 2012; 2(1): 2074-5346.

[4] European Food Safety Agency, EFSA. Opinion of the scientific panel on biological hazards on a request from the commission related to the use of antimicrobials for the control of Salmonella in poultry. The European Food Safety Agency Journal, 2004; 115: 1-76.

[5] Akoachere, J-F. T. K., Tanih, F. N., Ndip, L. M. \&Ndip, R. N. Phenotypic Characterization of Salmonella Typhimurium Isolates from Food-animals andAbattoir Drains in Buea, Cameroon. International centre for diarrhoeal disease research, Bangladesh Journal of Health and Population Nutrition, 2009; 27(5): 612-618

[6] Oluyege, J. O. and Oyinloye, I. A. Incidence of multiple antibiotic resistant among Salmonella spp. isolated from poultry droppings and cowdung in Ado-Ekiti metropolis. Researcher 201, 3;5(9) http://www.sciencepub.net/researcher

[7] Braden, C. R. Salmonellaentericaserotype Enteritidis and eggs: Anational epidemic in the United States. Clinical Infectious Disease, 2006; 43: 512-517.

[8] Linam, W. M. \& M. A. Gerber. Changing epidemiology and prevention of Salmonella infections. Pediatric Infectious Disease Journal, 2007; 26: 747-748

[9] Kabir, S. M. L. Avian colibacillosis and salmonellosis: A closer look at epidemiology, pathogenesis, diagnosis, control and public health concerns. International Journal of Environmental Research Public Health, 2010; 7: 89-114.

[10] WHO. Antimicrobial resistance. 2002; Fact Sheet No. 194 ed.

[11] Van, D. E., W. J. Wannet, D. J. Houwers, and W. Van Pelt. Antimicrobial susceptibilities of Salmonella strains isolated from humans, cattle, pigs, and chickens in the Netherlands from 1984 to 2001. Journal of Clinical Microbiology, 2003; 41: $3574-8$.

[12] Cailhol, J., et al. Trends in antimicrobial resistance phenotypes in non-typhoid Salmonellae from human and poultry origins in France. Epidemiology of Infections, 2006; 134: $171-180$.

[13] Alambedji, R. B., Fofana, M., Seydi, A. \&Akakpo, A. J. Antimicrobial Resistance of Salmonella isolated from poultry carcasses in Dakar (Senegal). Brazilian Journal of Microbiology, 2006; 37 (4): 57-61. 
[14] Okoli, I. C., G. E. Endujihe, \& I. P. Ogbuewu. Frequency of isolation of Salmonella from commercial poultry feeds and their antimicrobial resistance profiles, Imo State, Nigeria. Online Journal of Health and Allied Science, 2006; 5 (2): 3.

[15] Angulo, F. J., Baker, N. L., Olsen, S. J., Anderson, A.,Barett, T. J. Antimicrobial use in controlling the transfer of antimicrobial resistance to humans. Outbreak, 2004 Inc.

[16] Yoke-Kqueen, C., Learn-Han, L., Noorzaleha, A. S.,Son, R., Sabrina, S., Jiun-Horng, S. \& Chai-Hoon,K. Characterization of multiple-antimicrobialresistant Salmonella enterica subspecies enterica isolated from indigenous vegetables and poultry in Malaysia. Letters in Applied Microbiology, 2007; 46: 318-324.

[17] Pui, C. F., Wong, W. C., Chai, L. C., Lee, H. Y., Tang, J. Y. H., Noorlis, A., Farinazleen, M. G., Cheah, Y. K. \& Son, R. Biofilm formation by Salmonella Typhi and Salmonella Typhimurium on plastic cutting board and its transfer to dragon fruit. International Food Research Journal, 2011a; 18: 31-38.

[18] Begum, K., Reza, T. A., Hague, M., Hossain, A., Hassan, F. M. K., Hassan, S. N., Akhter, N., Ahmed, A. \&Barua, U. Isolation, Identification and antibiotic resistance pattern of Salmonella spp. from chicken eggs, intestines and environmental samples. Bangladesh Pharmaceutical Journal, 2010; 13: 23-27.

[19] Garba, A., Bolajoko, B. M.,.Barde, A. Ahmed, I., Sa'adatu, I. Agang, A. S.,Abdullahi, H. A., Bakari, U. I. J., Uraki, A. T., Abdurrahman, A.\& Goji, J. N. (2010). The threat of Salmonellosis to commercial poultry production in Adamawa state, Nigeria. Sokoto Journal of Veterinary Sciences, 2010; 8(1\&2): 50-52.

[20] Akond, M. A., A. Saidul, S. M. R. Hasan, and S. Momena. Distribution of Vibrio Cholerae and its Antibiotic Resistance in the Samples from Poultry and Poultry Environment of Bangladesh. Advances in Environmental Biology, 2009; 3 (1): 25-32.

[21] Clinical and Laboratory Standards Institute (CLSI). (2005). Performance standards for antimicrobial susceptibility testing; 15th informational supplement, CLSI/NCCLS M100-S15, Clinical and Laboratory Standards Institute, Wayne, PA.

[22] Cheesbrough M. (2006). District Laboratory Practice in Tropical Countries Part 2 Third Edition. Cambridge University Press. ISBN-13 978-0-521-67631-1
[23] Nogrady N, Toth A, Kostyak A, Paszti J, Nagy B. Emergence of multidrug-resistant clones of Salmonella Infantis in broiler chickens and humans in Hungary. Journal of Antimicrobial Chemotherapy, 2007; 60(3):645-8.

[24] Fallah R., Kiani A. \&Azarfar A. A review of the role of five kinds of alternatives to in feed antibiotics in broiler production Journal of Veterinary Medicine and Animal Health, 2013; 5: 317-321

[25] Crump, J. A. \& Mintz, E. D. Global trends in typhoid and paratyphoid fever. Emerging Infections, 2010; 50: 241-246.

[26] Khan, M., Ali, P., Suryanarayan M., Musthaq A., Reshma, B., Vaswani S. \& Mohammed, F. Antimicrobial susceptibility of Salmonella isolates from chicken meat samples in Dubai, United Arab Emirates. International Journal of Food, Nutrition and Public Health, 2010; 3(2).

[27] Zhao, S., Datta, A. R., Ayers, S., Friedman, S., Walker, R. D. \& White, D. G. Antimicrobial-resistant Salmonella serovars isolated from imported foods. International Journal of Food Microbiology 2003; 84(1): 87-92.

[28] Angulo, F. J., Nargund, V. N. \& Chiller, T. C. Evidence of an association between use of antimicrobial agents in food animals and anti-microbial resistance among bacteria isolated from humans and the human health consequences of such resistance. Journal of Veterinary Medicine. B, Infectious Diseases and Veterinary Public Health, 2004; 51, 374-379.

[29] Shah, A. H. \& Korejo, N. A. Antimicrobial Resistance Profile of Salmonella Serovars Isolated from Chicken Meat. Journal of Veterinary and Animal Science. 2012; 2: 40-46

[30] Musa, I. W., Mansur, M. S., Sa'idu, L., Mohammed, B., Kaltungo, B. Y., Lawan, M. K., Talba, A. M. Isolation and Antibiogram of Salmonella Species from Water and Poultry Feed In Selected Commercial Farms In Zaria, Nigeria. Time Journals of Agriculture and Veterinary Sciences, 2014; 2(2): $75-80$

[31] Martin, L. J., Murray, F., Doré, K., Buxton, J. A., Pollari,F., Henry, B., Middleton, D., Ahmed, R., Jamieson, F.,Gebin, B., McEwen, S. A. \& Wilson, J. B. The Multi-Provincial Salmonella Typhimurium Case Control Steering Committee. Increased burden of illnesses associated with antimicrobial resistant Salmonella enterica serotype typhimurium infections. Journal of Infectious Diseases, 2004; 189:377-384. 